\title{
Double Bennett Mechanisms with Assembly Modes of Different Dimensions
}

\author{
J.M. Selig ${ }^{1}$ and Z. $\mathrm{Li}^{2}$
}

\begin{abstract}
By considering the geometry of the Bennett 3R open-chain linkage it is possible to predict the possible configuration spaces of $6 \mathrm{R}$ closed loop mechanisms formed by joining pairs of Bennett linkages. Attention is confined to cases where the configuration space can be described by the intersection of three quadric surfaces in a three-dimensional projective space. There are just five possibilities which contain a component of dimension one. Two of these possibilities correspond to well-known constructions for forming mobile overconstrained 6R mechanisms from Bennett linkages. The geometry shows that these configuration spaces must also contain a number of isolated configurations, a result we believe to be new. Finally we are able to show that one of the other five geometric possibilities cannot occur as the configuration space of a double Bennett mechanism.
\end{abstract}

\section{INTRODUCTION}

It is well known that the molecular structure for cyclohexane $\left(C_{6} H_{12}\right)$ can be modeled by the line-symmetric Bricard mechanism. The molecule's carbon-carbon back-bone can be approximated by the $6 \mathrm{R}$ mechanism. The molecule has two conformational isomers, that is two possible assembly modes. These are known as the "boat" and "chair" configurations. As a mechanism the chair configuration is mobile but the boat is rigid, see [1]. The configuration space of this mechanism thus consists of two components with different dimensions, a one-dimensional mobile component and a zero-dimensional point.

In this work we find similar 6R mechanisms with assembly modes of different dimensions. In general the configuration space of these mechanisms is an algebraic variety defined as the intersection of a number of polynomial equations. Such an algebraic variety can have components of different dimensions only if it is not a complete intersection.

The overconstrained mechanisms that we construct are well-known combinations of Bennett mechanisms. However, we are able to give very simple derivations of the configuration spaces of the mechanisms based on intersecting planes and quadrics with the Study quadric. These derivations and the discovery of the isolated rigid assemblies is, we believe, novel.

\footnotetext{
${ }^{1}$ J.M. Selig is with the School of Engineering, London South Bank University, London SE1 0AA, U.K. seligjm@lsbu.ac.uk

${ }^{2} \mathrm{Z}$. $\mathrm{Li}$ is with the Institute of Discrete Mathematics and Geometry, Vienna University of Technology, Vienna, Austria zijia.li@tuwien.ac.at
}

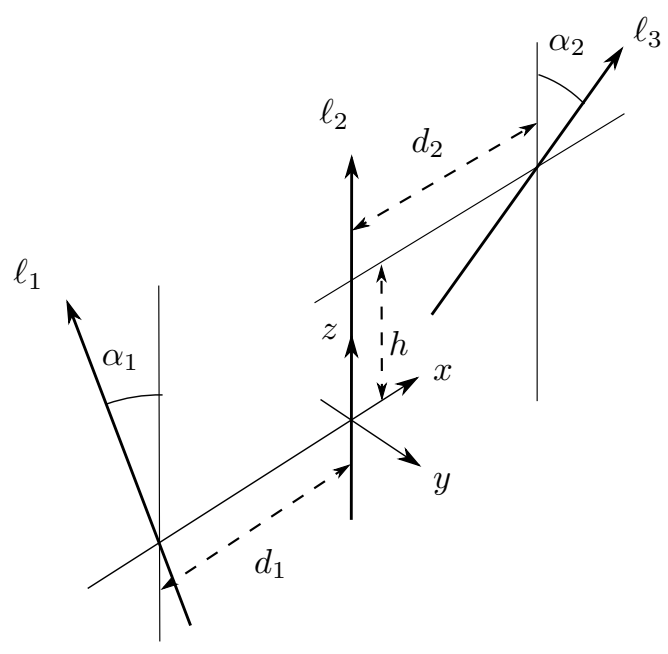

Fig. 1. The standard position for a general $3 R$ linkage.

\section{THE BENNETT LINKAGE}

In [9], it was shown that the Bennett linkage describes a three dimensional variety in the Study quadric. This variety is the projection of the Segre variety $\mathbb{P}^{1} \times \mathbb{P}^{1} \times \mathbb{P}^{1}$, and was shown to be the complete intersection of the Study quadric, a $\mathbb{P}^{5}$ and another quadric hypersurface.

The Study quadric is a six-dimensional quadric in $\mathbb{P}^{7}$, writing the homogeneous coordinates of the $\mathbb{P}^{7}$ as $\left(a_{0}: a_{1}\right.$ : $\left.a_{2}: a_{3}: c_{0}: c_{1}: c_{2}: c_{3}\right)$, the equation of the Study quadric is given by,

$$
a_{0} c_{0}+a_{1} c_{1}+a_{2} c_{2}+a_{3} c_{3}=0 .
$$

Now consider the standard or home position for a general 3R linkage shown in Fig. 1. The Bennett conditions are imposed by requiring that $h=0$ and

$$
\frac{d_{1}}{\sin \alpha_{1}}=\frac{d_{2}}{\sin \alpha_{2}}=d,
$$

where $d$ is this common ratio sometimes called the Bennett ratio of the linkage, see [6]. In fact there are two possible forms for the Bennett condition $d_{1} / \sin \alpha_{1}=d_{2} / \sin \alpha_{2}$, as above, and $d_{1} / \sin \alpha_{1}=-d_{2} / \sin \alpha_{2}$. Both the equations give the Bennett condition when $h=0$.

We can eliminate $d_{1}$ and $d_{2}$ now since,

$$
d_{1}=d \sin \alpha_{1}, \quad \text { and } \quad d_{2}=d \sin \alpha_{2}
$$

when the Bennett conditions are satisfied. 
With the coordinates given in Fig. 1 , the $\mathbb{P}^{5}$ that the constraint variety lies on is determined by a pair of hyperplanes,

$$
\begin{aligned}
d \sin \left(\alpha_{1}+\alpha_{2}\right) a_{2}-\left(\sin \alpha_{1}\right. & \left.+\sin \alpha_{2}\right) c_{2} \\
& -\left(\cos \alpha_{1}-\cos \alpha_{2}\right) c_{3}=0 \\
\left.d\left(\sin \alpha_{1}+\sin \alpha_{2}\right)\right) a_{1}+\left(\operatorname { c o s } \left(\alpha_{1}\right.\right. & \left.\left.+\alpha_{2}\right)-1\right) c_{0} \\
& -\sin \left(\alpha_{1}+\alpha_{2}\right) c_{1}=0
\end{aligned}
$$

see [9]. The explicit form of the other quadric hypersurface was not found in [9], we look at this now.

The standard Segre variety $\mathbb{P}^{1} \times \mathbb{P}^{1} \times \mathbb{P}^{1}$ satisfies the 9 quadrics, in a $\mathbb{P}^{7}$. Writing the homogeneous coordinates of this $\mathbb{P}^{7}$ as $\left(X_{0}: X_{1}: X_{2}: X_{3}: Y_{0}: Y_{1}: Y_{2}: Y_{3}\right)$ the 9 quadrics can be written,

$$
\begin{aligned}
& Q_{1}: X_{0} Y_{1}+X_{2} X_{3}=0, \\
& Q_{2}: X_{0} Y_{2}-X_{1} X_{3}=0, \\
& Q_{3}: X_{0} Y_{3}+X_{2} X_{3}=0 \\
& Q_{4}: X_{1} X_{0}+Y_{2} Y_{3}=0, \\
& Q_{5}: X_{2} Y_{0}-Y_{1} Y_{3}=0, \\
& Q_{6}: X_{3} Y_{0}+Y_{1} Y_{2}=0, \\
& Q_{7}: X_{0} Y_{0}+X_{1} Y_{1}=0, \\
& Q_{8}: X_{0} Y_{0}-X_{2} Y_{2}=0, \\
& Q_{9}: X_{0} Y_{0}+X_{3} Y_{3}=0,
\end{aligned}
$$

see [9] and theorem 3 in [6]. The general 3R linkage determines a transformed version of this variety. The transformation is given by the matrix $M$ such that,

$$
\left(\begin{array}{l}
a_{0} \\
a_{1} \\
a_{2} \\
a_{3} \\
c_{0} \\
c_{1} \\
c_{2} \\
c_{3}
\end{array}\right)=M\left(\begin{array}{l}
X_{0} \\
X_{1} \\
X_{2} \\
X_{3} \\
Y_{0} \\
Y_{1} \\
Y_{2} \\
Y_{3}
\end{array}\right)
$$

Where the columns of $M$ are given by, $1, \ell_{1}, \ell_{2}, \ldots,-\ell_{1} \ell_{2}$, where $\ell_{i}$ is the dual quaternion representing the axis of the $i$ th joint. The (transposed) columns of $M$ are ,

$$
\begin{aligned}
1^{T}= & (1,0,0,0,0,0,0,0) \\
\ell_{1}^{T}= & \left(0,0, \sin \alpha_{1}, \cos \alpha_{1}, 0,0, d_{1} \cos \alpha_{1},-d_{1} \sin \alpha_{1}\right) \\
\ell_{2}^{T}= & (0,0,0,1,0,0,0,0) \\
\ell_{3}^{T}= & \left(0,0,-\sin \alpha_{2}, \cos \alpha_{2}, 0, h \sin \alpha_{2},\right. \\
\left(\ell_{1} \ell_{2} \ell_{3}\right)^{T}= & \left(0,0, \sin \left(\alpha_{2}-\alpha_{1}\right),-\cos \alpha_{2},-d_{2} \sin \alpha_{2}\right) \\
& -h \sin \alpha_{1} \sin \alpha_{2},-h \cos \alpha_{1} \sin \alpha_{2}, \\
& \left.\left(d_{2}-d_{1}\right) \cos \left(\alpha_{2}-\alpha_{1}\right),\left(d_{2}-d_{1}\right) \sin \left(\alpha_{2}-\alpha_{1}\right)\right) \\
-\left(\ell_{2} \ell_{3}\right)^{T}= & \left(\cos \alpha_{2},-\sin \alpha_{2}, 0,0,-d_{2} \sin \alpha_{2},-d_{2} \cos \alpha_{2},\right. \\
\left(\ell_{1} \ell_{3}\right)^{T}= & \left(-\cos \left(\alpha_{1}+\alpha_{2}\right), \sin \left(\alpha_{1}+\alpha_{2}\right), 0,0,\right. \\
& \left(d_{1}+d_{2}\right) \sin \left(\alpha_{1}+\alpha_{2}\right),\left(d_{1}+d_{2}\right) \cos \left(\alpha_{1}+\alpha_{2}\right), \\
& \left.h \cos \alpha_{1} \sin \alpha_{2},-h \sin \alpha_{1} \sin \alpha_{2}\right) \\
-\left(\ell_{1} \ell_{2}\right)^{T}= & \left(\cos \alpha_{1},-\sin \alpha_{1}, 0,0,-d_{1} \sin \alpha_{1},\right. \\
&
\end{aligned}
$$

Now the matrix $M$ is singular if the Bennett conditions are imposed. So let us simplify thing a little by imposing,

$$
d_{1}=d \sin \alpha_{1}, \quad \text { and } \quad d_{2}=d \sin \alpha_{2}
$$

but not $h=0$. It is also possible to do this calculation the other way around, setting $h=0$ but not $d_{1} / \sin \alpha_{1}=$ $d_{2} / \sin \alpha_{2}$ until the end. Geometrically this can be interpreted as defining a family of maps, parameterised by $h$. For each $h$ the map is invertible but when $h=0$ the inverse is the closure of the map. A more prosaic way to look at this is to consider the the inverse as given by $M^{-1}=\frac{1}{\operatorname{det}(M)} \operatorname{Adj}(M)$ where $\operatorname{Adj}(M)$ denotes the adjugate of $M$. Now since the corank of $M$ is 2 , both the determinant and adjugate become zero when $h=0$. This is essentially an indeterminate form and we can take the limit as $h \rightarrow 0$ by dividing $\operatorname{Adj}(M)$ by $h$ and then setting $h=0$. This gives a matrix,

$$
\left(\frac{\operatorname{Adj}(M)}{h}\right)_{h=0}
$$

whose entries can be computed explicitly. However there isn't enough space to write this out in full. Rather, consider,

$$
\left(\begin{array}{l}
X_{0} \\
X_{1} \\
X_{2} \\
X_{3} \\
Y_{0} \\
Y_{1} \\
Y_{2} \\
Y_{3}
\end{array}\right)=\left(\frac{\operatorname{Adj}(M)}{h}\right)_{h=0}\left(\begin{array}{l}
a_{0} \\
a_{1} \\
a_{2} \\
a_{3} \\
c_{0} \\
c_{1} \\
c_{2} \\
c_{3}
\end{array}\right) .
$$

Now we can write,

$$
\begin{aligned}
& X_{0}=d\left(\sin \alpha_{1}-\sin \alpha_{2}\right) a_{2}+ \\
& \sin \left(\alpha_{2}-\alpha_{1}\right) c_{2}+\left(1-\cos \left(\alpha_{1}-\alpha_{2}\right)\right) c_{3}, \\
& X_{1}=d \sin \left(\alpha_{2}-\alpha_{1}\right) a_{1}+ \\
& \left(\cos \alpha_{2}-\cos \alpha_{1}\right) c_{0}+\left(\sin \alpha_{1}-\sin \alpha_{2}\right) c_{1}, \\
& X_{2}=-d\left(\sin \alpha_{1}+\sin \alpha_{2}\right) a_{1}+ \\
& \left(1-\cos \left(\alpha_{1}+\alpha_{2}\right)\right) c_{0}+\sin \left(\alpha_{1}+\alpha_{2}\right) c_{1}, \\
& X_{3}=d \sin \left(\alpha_{1}-\alpha_{2}\right) a_{1}+ \\
& \left(\cos \alpha_{1}-\cos \alpha_{2}\right) c_{0}+\left(\sin \alpha_{2}-\sin \alpha_{1}\right) c_{1}, \\
& Y_{0}=-d\left(\sin \alpha_{1}+\sin \alpha_{2}\right) a_{1}+ \\
& \left(1-\cos \left(\alpha_{1}+\alpha_{2}\right)\right) c_{0}+\sin \left(\alpha_{1}+\alpha_{2}\right) c_{1}, \\
& Y_{1}=-d \sin \left(\alpha_{1}+\alpha_{2}\right) a_{2}+ \\
& \left(\sin \alpha_{1}+\sin \alpha_{2}\right) c_{2}+\left(\cos \alpha_{1}-\cos \alpha_{2}\right) c_{3}, \\
& Y_{2}=d\left(\sin \alpha_{1}-\sin \alpha_{2}\right) a_{2}+ \\
& \sin \left(\alpha_{2}-\alpha_{1}\right) c_{3}+\left(1-\cos \left(\alpha_{1}-\alpha_{2}\right)\right) c_{3}, \\
& Y_{3}=d \sin \left(\alpha_{1}+\alpha_{2}\right) a_{2}- \\
& \left(\sin \alpha_{1}+\sin \alpha_{2}\right) c_{2}+\left(\cos \alpha_{2}-\cos \alpha_{1}\right) c_{3} .
\end{aligned}
$$

A few other common factors have been cancelled in the above.

This can be used to substitute for the coordinates $X_{0}, \ldots, Y_{3}$ in the nine quadrics (2). From the computations it turns out that if we do this $Q_{7}, Q_{8}$ and $Q_{9}$ vanish. We 
also get that $Q_{6}=Q_{1}$ and $Q_{3}=Q_{4}=-Q_{1}$. And finally that,

$$
Q_{1}\left(\sin \alpha_{1}+\sin \alpha_{2}\right)+Q_{5} \sin \left(\alpha_{1}-\alpha_{2}\right)=0,
$$

and

$$
Q_{1} \sin \left(\alpha_{1}-\alpha_{2}\right)+Q_{2}\left(\sin \alpha_{1}+\sin \alpha_{2}\right)=0 .
$$

So all the quadrics vanish or are multiples of $Q_{1}$ (say). As an $8 \times 8$ matrix this quadric can be written,

$$
Q_{1}=\left(\begin{array}{cccccccc}
0 & 0 & 0 & 0 & 0 & 0 & 0 & 0 \\
0 & 2 d^{2} \kappa_{1} & 0 & 0 & d \kappa_{4} & d \kappa_{5} & 0 & 0 \\
0 & 0 & 2 d^{2} \kappa_{2} & 0 & 0 & 0 & d \kappa_{6} & d \kappa_{8} \\
0 & 0 & 0 & 0 & 0 & 0 & 0 & 0 \\
0 & d \kappa_{4} & 0 & 0 & 2 \kappa_{3} & \kappa_{7} & 0 & 0 \\
0 & d \kappa_{5} & 0 & 0 & \kappa_{7} & 2 \kappa_{2} & 0 & 0 \\
0 & 0 & d \kappa_{6} & 0 & 0 & 0 & 2 \kappa_{1} & \kappa_{9} \\
0 & 0 & d \kappa_{8} & 0 & 0 & 0 & \kappa_{9} & 2 \kappa_{10}
\end{array}\right),
$$

where,

$$
\begin{array}{ccc}
\kappa_{1}= & -\sin \left(\alpha_{1}-\alpha_{2}\right)\left(\sin \alpha_{1}+\sin \alpha_{2}\right) \\
\kappa_{2}= & -\left(\sin \alpha_{1}-\sin \alpha_{2}\right) \sin \left(\alpha_{1}+\alpha_{2}\right) \\
\kappa_{3}= & \left(\cos \alpha_{1}-\cos \alpha_{2}\right)\left(1-\cos \left(\alpha_{1}+\alpha_{2}\right)\right) \\
\kappa_{4}= & \left(\sin \left(\alpha_{1}-\alpha_{2}\right)\left(1-\cos \left(\alpha_{1}+\alpha_{2}\right)\right)-\right. \\
& \left.\left(\sin \alpha_{1}+\sin \alpha_{2}\right)\left(\cos \alpha_{1}-\cos \alpha_{2}\right)\right) \\
\kappa_{5}= & \left(\left(\sin \alpha_{1}-\sin \alpha_{2}\right)\left(\sin \alpha_{1}+\sin \alpha_{2}\right)+\right. \\
\kappa_{6}= & \left(\left(\sin \alpha_{1}-\sin \alpha_{2}\right)\left(\sin \alpha_{1}+\sin \alpha_{2}\right)-\right. \\
\kappa_{7}= & \left(\sin \left(\alpha_{1}+\alpha_{2}\right)\left(\cos \alpha_{1}-\cos \alpha_{2}\right)-\right. \\
\kappa_{8}= & \left(\left(\sin \alpha_{1}-\sin \alpha_{2}\right)\left(\cos \alpha_{1}-\cos \alpha_{2}\right)-\right. \\
\kappa_{9}= & \left(\left(1-\cos \left(\alpha_{1}-\alpha_{2}\right)\right)\left(\sin \alpha_{1}+\sin \alpha_{2}\right)+\right. \\
\kappa_{10}= & \left(1-\cos \left(\alpha_{1}-\alpha_{2}\right)\right)\left(\cos \alpha_{1}-\cos \alpha_{2}\right) \\
& \left.\sin \left(\alpha_{1}-\alpha_{2}\right)\left(\cos \alpha_{1}-\cos \alpha_{2}\right)\right)
\end{array}
$$

Notice that the quadric $Q_{1}$ is singular along the line $a_{1}=$ $a_{2}=c_{0}=c_{1}=c_{2}=c_{3}=0$. Moreover, this line singularity lies in the $\mathbb{P}^{5}$ determined by the equations in (1).

\section{MOBILE DOUBLE BENNETT MECHANIMS}

The rigid-body displacements generated by a Bennett $3 \mathrm{R}$ open-loop linkage is then the intersection of the Study quadric with a $\mathbb{P}^{5}$ and another quadric. Suppose now that we join two Bennett 3R linkages to form a single 6R loop, in general the position and orientation of the coupler bar will be given by the intersection of two $\mathbb{P}^{5}$ s giving a $\mathbb{P}^{3}$ and three quadrics, the Study quadric and two others. That is, the intersection of 3 quadrics in a $\mathbb{P}^{3}$. Generally, that is when the intersection is complete, there will be $2^{3}=8$ points of intersection giving 8 different assembly configurations or modes. So in general, no mobile components. Here we are interested in the special cases where the intersection contains a component with dimension one.

If the two $\mathbb{P}^{5} \mathrm{~s}$ meet exceptionally in a $\mathbb{P}^{4}$ then the 6R single loop, mechanism will be mobile. These cases, including the possible degenerate sub-cases, were discussed by Dietmaier [4], so will not be discussed further here.

Assuming the two 5-planes meet in a 3-plane, there are only 5 possibilities for a mobile mechanism. To see this consider the intersection of only two of the quadrics in the $\mathbb{P}^{3}$, this will be a curve of degree 4 . The general case will be an elliptic quartic curve, but such a curve cannot lie in the third quadric unless this quadric is a linear combination of the first two. However, the quartic curve can degenerate, it may split into; four lines, two lines and a conic, two conics or a line and a twisted cubic curve. Now some of these components may lie in the third quadric. The possibilities are that the three quadrics share a single line, a pair of lines, a conic, a conic and a line or a twisted cubic curve.

\section{A. A Line and 4 Points}

If the intersection of the three quadrics in $\mathbb{P}^{3}$ contain a single line then the intersection will also contain 4 isolated points. There are two ways to show this. First consider the intersection of two of the quadrics, say the Study quadric and one of the Bennett quadrics. If the intersection contains a line then the intersection must also contain a residual curve which in general will be a twisted cubic. The same must be true for the intersection of the second Bennett quadric with the Study quadric, we assume the line will be the same but the residual cubic will be different. The intersection of the Study quadric with the $\mathbb{P}^{3}$ will generally be a non-singular 2 dimensional quadric. The two twisted cubics must lie in this quadric surface and each will have bi-degree $(1,2)$, they both meet the same line in the quadric in two points. The intersection of these two cubics will consist of $1 \times 2+2 \times 1=$ 4 points. Alternatively, consider the intersection of two of the quadrics in the $\mathbb{P}^{3}$ again. The intersection is assumed to be a line and a twisted cubic curve as before. Now intersect this with the third quadric.We assume that the line lies in this quadric. The intersection of the twisted cubic with the quadric will consist of $2 \times 3=6$ points. However, two of these points must be where the twisted cubic meets the line, hence there will be $6-2=4$ other points of intersection.

The arguments above apply to three quadrics in a $\mathbb{P}^{3}$, but are there double Bennett mechanisms that give this geometry? Notice that in the Study quadric a line corresponds to the subgroup of rotations about a fixed line or the subgroup of translations parallel to a fixed direction. Actually, this describes lines through the identity in the group, a general line in the Study quadric is one of these subgroups multiplied by a constant displacement. It is clear that the $3 \mathrm{R}$ Bennett linkage cannot perform pure translations, otherwise there would be a mobile $3 R P$ mechanism. We can have a $3 R$ Bennett linkage where the last link rotates about a fixed line, this is just the $4 \mathrm{R}$ Bennett loop. The question now arises, which lines can the end-effector of the $3 R$ Bennett linkage 


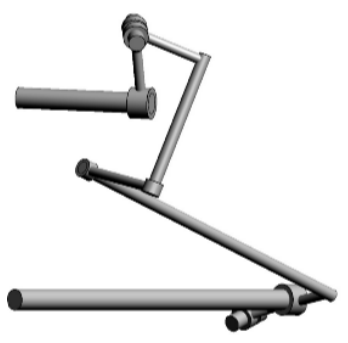

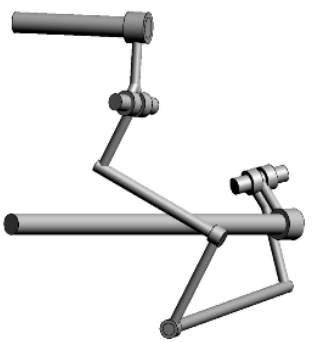
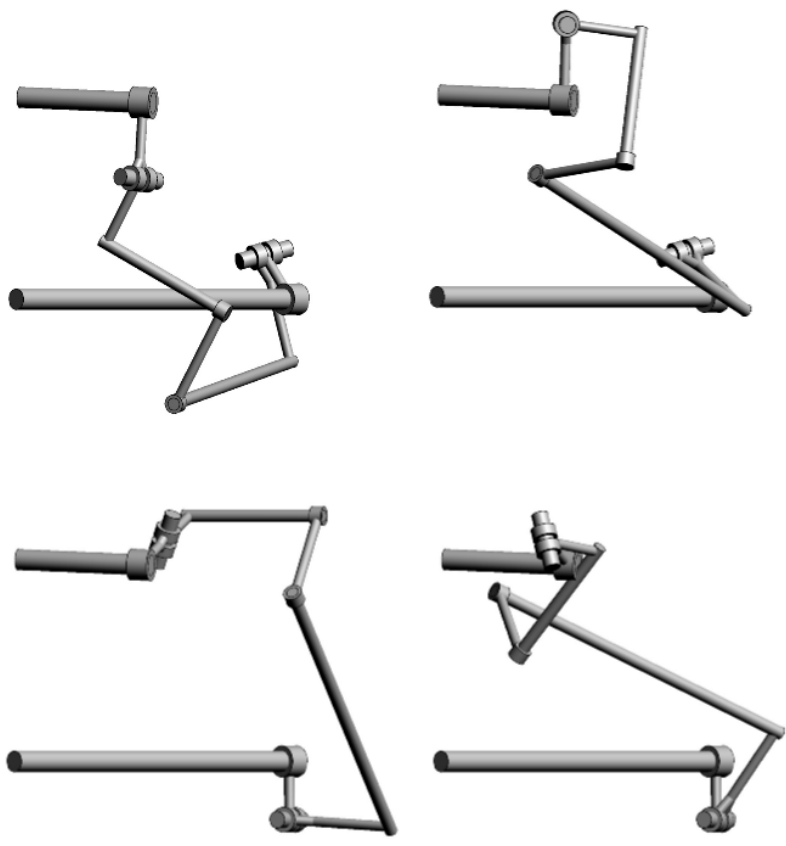

Fig. 2. A Double Bennett Maechanism with One Mobile Assembly and Four Isolated Assemblies.

rotate about? Notice that the first joint of the $3 \mathrm{R}$ linkage is fixed, so the virtual 4th joint of a Bennett mechanism can lie on the regulus traced out when the 4th joint is rotated about the first joint. Now suppose we take two Bennett $4 \mathrm{R}$ mechanisms and join them in such a way that the axis of the 4th joint in each coincides. We then join the third joints of both mechanisms with a rigid link. This combined mechanism is clearly still mobile, the new link simply rotates about the combined 4 th joint. Finally we can remove the 4th joint and all the links attached to it, the result will be a mobile 6R mechanism.

This construction seems to be due to Bennett [10]. The results is an overconstrained mobile mechanism known as the Waldron Hybrid mechanism. From the above we see that the configuration space of the mechanism consists of a line and 4 points. Note that the algebraic geometry above assumes complex scalars but we are really only interested in points with real coordinates. So we are left with the question as to whether or not all 4 isolated points in the variety defined by the linkage can be real assembly configurations. This can be answered with an example, fig. 2 shows a 6R double Bennett mechanism with a mobile assembly mode and 4 real isolated assemblies. The Denavit-Hartenberg parameters for this mechanism are,

Twist angles $1.94,2.04,2.79,0.72,1.55,1.99$ radians, Link lengths $1.79,1.83,4.82,1.39,2.64,2.56$,

$$
\text { Offsets } \quad 0,-2.85,-9.05,0,-8.33,-3.29 \text {. }
$$

\section{B. Conic and Two Points}

If the three quadrics in $\mathbb{P}^{3}$ defining the configuration space of the $6 \mathrm{R}$ double Bennett mechanism contain a conic curve then the intersection will also contain a pair of isolated points (or a line, see below). This can be seen using the same methods as in the previous section. Consider again the 2dimensional quadric which is the intersection of the Study quadric with the $\mathbb{P}^{3}$. The intersection of this with the quadric determined by one of the Bennett linkages will be a pair of conic curves. Now a conic must be a complete intersection and so its bi-degree in the 2-dimensional quadric must be $(1,1)$. This also applies to the intersection of the other quadric with the 2-dimensional quadric. The intersection of two $(1,1)$ curves in a quadric surface gives $(1 \times 1)+(1 \times 1)=$ 2 points.

We can produce a double Bennett mechanism whose configuration space contains a conic using another well known construction. It is well known that the configuration space of a $4 \mathrm{R}$ Bennett mechanism is conic curve. That is the coupler bar of the Bennett mechanism traces out a conic curve in the Study quadric, see [3]. Now we can attach a 3R Bennett linkage to either side of a closed loop Bennett mechanism. That is, the final joint of the linkage coincides with either the second or third joint in the closed loop mechanism. So long as the Bennett conditions are satisfied for each new loop formed, the resulting mechanism will be mobile. Finally we remove all of the original closed loop mechanism apart from the second and third joint and the coupler link connecting these two joints. This construction give a Goldberg primary 6R mechanism, see [2]. Clearly by construction, the configuration space of the mechanism contains a conic in the Study quadric, the coupler curve of the original closed-loop Bennett mechanism the construction was based on. The above shows however, that the configuration space of the curve can also 

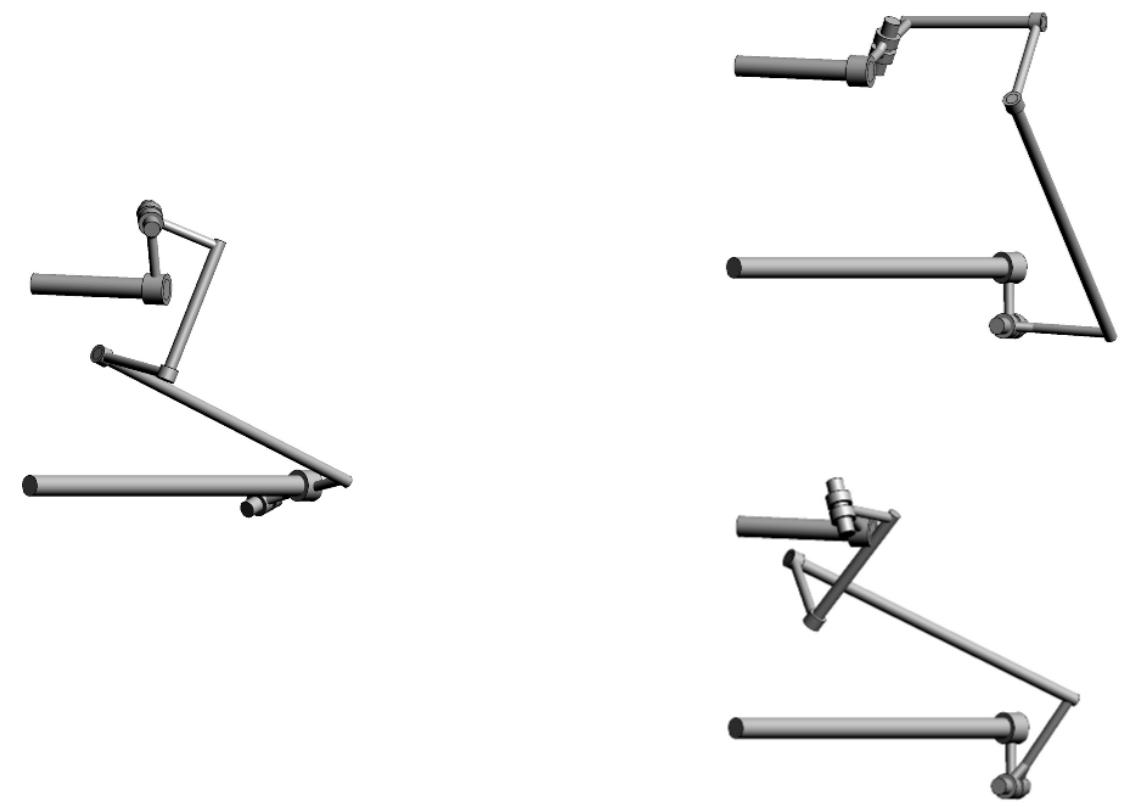

Fig. 3. A Double Bennett Maechanism with One Mobile Assembly and Two Isolated Assemblies.

contain up to two real isolated configurations. The example shown in fig. 3 shows that both isolated points can be real. The Denavit Hartenberg parameters for this mechanism are,

Twist angels $1.94,1.24,1.99,1.65,1.97,1.99$ Radians, Link lengths $1.79,1.83,0.76,1.79,1.95,2.14$,

Offsets $\quad 0,-2.55,2.55,0,-2.25,-2.25$.

\section{Two lines and Two points}

If three quadrics in $\mathbb{P}^{3}$ contain a pair of lines, then the intersection will also contain two isolated points. This can be seen using the same argument as in the previous section. In this case however, there is no double Bennett mechanism with this geometry.

To see this consider the construction outlined in section III-A above. Think of the two 3R Bennett linkages as a left linkage and a right linkage joined at the coupler link. Suppose the virtual fourth joint shared by the two Bennett mechanisms is $\ell$. This common line $\ell$ must lie on two reguli, one generated by swinging $\ell$ about the first joint in the left linkage and the other generated by turning $\ell$ about the first joint in the right linkage. Now it is possible for two cylindrical reguli to intersect in a pair of lines.

However, for the $6 \mathrm{R}$ mechanism to exist with these two mobile modes we must be able to disassemble the mechanism and reassemble it in the other mode. Suppose the mechanism is mobile in one mode, the coupler rotating about the virtual 4th joint $\ell$. Now disassemble the mechanism by splitting it at the final joint of the left Bennett linkage. To reassemble the mechanism in the other mode we must subject the coupler link to a rigid-body displacement. To move the last link in the left linkage to its new position without changing the geometry of the mobile virtual Bennett mechanism, all we can do is to rotate all the links and joints about the first joint of the linkage. For the right linkage we have a little more freedom, we can rotate about the first joint of the right linkage but we can also rotate about the final link in the right linkage since this will only move the coupler link. The displacement in the right linkage must be the same as the displacement in the left linkage. We don't need a continuous family of solutions, just a single solution will do. However, it is not difficult to see that, apart from trivial solutions, the only way that a rotation about one line can be equal to a pair of rotations about two other lines is if the lines are all parallel or all coincident. None of these solutions gives a double Bennett mechanism. For a more formal proof of this claim consider the screw triangle formed by the three rotations. Since these are all rotations each can be decomposed into a pair of half-turns about lines perpendicular to their rotation axes and separated by half their rotation angles. For one rotation to be equal to the product of the other two, the axes of the half-turns must form a triangle which implies that the three rotation axes of the original rotations must be parallel. The exceptions are where all the axes meet at a point or all the rotation angles are zero.

\section{CONCLUSIONS}

This is not the only way that a mobile mechanism could have a configuration space that contains an isolated point. Suppose that the configuration space contains a complex component. The points of such a component are not physical and so would not be valid configurations of the real mechanism. It is possible however, that such a complex component 
contains a real singularity. That is a singular point with real coordinates. An example of this phenomenon occurs for a planar four bar where the sum of the three shortest bars equals the length of the longest bar. This clearly has a single, singular configuration. Presumably, the equations for this mechanism can be set up so that this configuration is a real singularity on a one-dimensional complex variety.

In future work we hope to be able to settle the two other cases, the twisted cubic and the case where the configuration space of the mechanism is a line and a conic. However, it seems likely that, as with the case of two lines and two points, double Bennett mechanisms with these configuration spaces cannot occur. This is, of course, only speculation at the moment.

A complete classification of $6 \mathrm{R}$ Bennett-based overconstrained mechanisms is not known, as far as we are aware. The Bennett-based mechanisms form a much larger class than the double Bennett mechanism considered here. For example, a mobile $6 \mathrm{R}$ can be constructed as an intersection of a Bennett $3 \mathrm{R}$ and a spherical or planar 3R, this is well-known in the literature. The Wohlhart-partial-symmetric $6 \mathrm{R}$ is another example which can be constructed as an intersection of a Bennett $3 R$ and a general $3 R$ linkage. In [11] four Bennett mechanisms are combined, then a central joint and its 4 links are removed, freezing two more joints can produce mobile overconstrained 6R mechanism. All four cases were studied Dietmaier [4]. Only one of the cases is a double Bennett mechanism as studied here, this is Wohlhart's double-Goldberg 6R linkage. Another double Bennett mobile $6 \mathrm{R}$ is discussed in [5]. This is also a combination of two Goldberg linkages but gives a different mechanism to Wohlhart's construction.

Another combination of two Bennett loops can be found in [7] and [8]. These papers focus on 7R mechanisms which are constructed by combining two Bennett loops sharing one joint.

\section{REFERENCES}

[1] J. Eddie Baker, 1986, "Limiting Positions of a Bricard Linkage and their Possible Relevance to the Cyclohexane molecule", Mechanism and Machine Theory, 21(3):253-260.

[2] J. Eddie Baker, 1993, "A Comparative Survey of the Bennett-Based, 6-Revolute Kinematic Loops", Mechanism and Machine Theory, 28(1):83-?96.

[3] O. Bottema and B. Roth, 1990, Theoretical Kinematics, Dover Publications, New York.

[4] P. Dietmaier, 1995, "Einfach übergeschlossene Mechanismen mit Drehgelenken", Habilitation thesis, Graz University of Technology, Graz, Austria

[5] Z. Li, 2014, "Sharp linkages", In: Lenarčič J., Khatib O. (eds) Advances in Robot Kinematics. Springer, Cham, Chapter 15, pp:131138.

[6] G. Hegedüs, Z. Li, J. Schicho and H-P. Schröcker, 2015, "The theory of bonds II: Closed 6R linkages with maximal genus", Journal of Symbolic Computation, 68(2), pp.167-180.

[7] M. Pfurner, X. Kong and C. Huang, 2014, "Complete kinematic analysis of single-loop multiple-mode 7-link mechanisms based on Bennett and overconstrained RPRP mechanisms", Mechanism and Machine Theory, 73:117-129.

[8] M. Pfurner and X. Kong, 2017, "Algebraic analysis of a new variableDOF 7R mechanism", author=Pfurner, Martin and Kong, Xianwen, In: P. Wenger and P. Flores (eds) New Trends in Mechanism and Machine Science, Springer, Cham, Chapter 8, pp:71-79.

[9] J.M. Selig, 2014, "Some Remarks on the RRR Linkage." In: Lenarčič J., Khatib O. (eds) Advances in Robot Kinematics. Springer, Cham, Chapter 9, pp:77-85.

[10] K. Waldron, 1968, "Hybrid Overconstrained Linkages" J. Mechanisms, 3:73-78.

[11] Chao-Yang Song, Huijuan Feng, Yan Chen, I-Ming Chen, Rongjie Kang, 2015, "Reconfigurable mechanism generated from the network of Bennett linkages", Mechanism and Machine Theory, 88:49-62. 